\title{
Experimental evaluation of the uplink dynamic range threshold
}

\author{
Sascha Berger ${ }^{1 *}$, Martin Danneberg ${ }^{1}$, Paolo Zanier ${ }^{2}$, Ingo Viering ${ }^{3}$ and Gerhard Fettweis ${ }^{1}$
}

\begin{abstract}
Despite the orthogonality of LTE's uplink transmission, it can happen that signals which are received at the base station with low power are drown out by strong signals from other users. Reason is the limited quantization resolution of the base station's analog-to-digital converter. Since this effect causes a quality of service degradation for users at the cell edge, the dynamic receive power range of uplink signals arriving at the base station should be upper limited. However, setting an adequate upper limit for the dynamic receive power range is not trivial since many effects, such as quantization noise, fast fading etc., decrease the dynamic range which the analog-to-digital converter can handle in theory. In this paper, we measure the maximal uplink dynamic receive power range, denoted as uplink dynamic range threshold, for our LTE-like measurement setup by monitoring the uplink bit-error-rate of a first user while decreasing its transmit power during the presence of a second adjacently scheduled user with fixed transmit power. As the measured threshold is $31.4 \pm 7.6 \mathrm{~dB}$, we can conclude that a link-budget-based estimation of the dynamic receive power range, which has been published previously, is accurate since its result coincides with the measurement.
\end{abstract}

Keywords: Uplink; Dynamic range; LTE; Transmit power control; Analog-to-digital converter

\section{Introduction}

A major feature of Long Term Evolution (LTE) Uplink (UL) is the Single Carrier Frequency Division Multiple Access (SC-FDMA) transmission scheme which separates signals from different user equipments (UEs) by frequency and, therefore, avoids frequency overlap between different signals. However, the near-far problem is still existent in the LTE UL. Reason is that the signals from all UEs that are received at the same time have to jointly pass the analog receiver front-end, which includes the analog-todigital converter (ADC), before the user separation takes place. If a UE's received signal is much stronger than the one of another UE, then the weak signal can be drowned out by the strong signal due to the limited quantization resolution of the ADC [1]. Since the difference in path loss in a LTE macro cell can easily be in the range of $60 \mathrm{~dB}$, we are forced to control the UE's transmit power in order to guarantee that all signals received at the base station (BS) at the same time are within a certain range. The range of received signal powers at the BS is referred

*Correspondence: sascha.berger@tu-dresden.de

${ }^{1}$ Vodafone Chair Mobile Communcations Systems, Technische Universität Dresden, 01062 Dresden, Germany

Full list of author information is available at the end of the article to as the UL dynamic receive power range (DR) $D$ and is defined as the difference between the 5th and the 95th percentile of the set of receive powers (in $\mathrm{dB}$ ) of signals that are received at the $\mathrm{BS}$ simultaneously $[2,3]$. The DR at which the weak UEs start to suffer performance degradation ${ }^{1}$ depends on the quantization resolution of the ADC and is denoted as the DR threshold $D_{\mathrm{TH}}$. LTE's UL transmit power control (TPC) [4] enables adjusting the UE's transmit power according to its path loss perceived and, therefore, is an adequate solution for limiting DR. Considering novel techniques such as carrier aggregation, we expect that the total bandwidth and the number of signals to be processed simultaneously by the ADC will increase in future. Hence, the problem of keeping DR below the DR threshold is expected to intensify and, therefore, one of the challenges future mobile networks are facing.

Bulakci et al. consider DR in [2] when optimizing the TPC in a relay-based LTE-advanced heterogeneous network. They avoid DRs above $20 \mathrm{~dB}$, i.e., they set $D_{\mathrm{TH}}=$ $20 \mathrm{~dB}$. However, Bulakci et al. do not justify this choice. In [3], Berger et al. derive a closed form expression for DR as a function of LTE's TPC parameters $\alpha$ (the path loss correction factor) and $P_{0}$ (a cell/UE specific parameter) and estimate the DR threshold to be in the range from 20 to

\section{黑 Springer}

C 2015 Berger et al. Open Access This article is distributed under the terms of the Creative Commons Attribution 4.0 International License (http://creativecommons.org/licenses/by/4.0/), which permits unrestricted use, distribution, and reproduction in any medium, provided you give appropriate credit to the original author(s) and the source, provide a link to the Creative Commons license, and indicate if changes were made. 
$50 \mathrm{~dB}$. They derive these numbers by considering the link budget of ADCs with a resolution between 13 bits and 16 bits $^{2}$. However, to the best knowledge of the authors of this work, there is neither an experimental nor a theoretical contribution published that provides a precise value for the DR threshold given a certain ADC. Surely, knowing the DR threshold is important in order to be able to configure the TPC such that the actual DR present at the BS is smaller than the BS's DR threshold.

Hence, in this work, we experimentally determine the DR threshold for an ADC with 12 bits quantization resolution and compare our result with the link-budget-based estimations from [3]. We arrange an experimental setup that closely mimics the LTE UL transmission of two UEs to a single BS. By holding the bandwidth and the channel constant for both UEs, we can adjust the signal strength of which the UEs are received at the BS by adjusting the UE's transmit powers. In this way, we can realize any arbitrary difference in the receive powers between the two UEs. By observing the weak UE's bit error rate (BER), we can define the receive power difference at which the weak UE's signal is drown out by the strong UE's signal. Based on this measurement, we can compute the DR threshold. Furthermore, we show that the interference scenario has an impact on the resulting DR threshold as we are using BER measurements to identify when the weak UE is drown out by the strong UE. We estimate the error of the DR threshold measurement and compare it to a link-budget-based estimation.

\section{Theoretical background}

This section is dedicated to provide theoretical background about LTE's open-loop UL TPC, DR, and the DR threshold.

\subsection{LTE open-loop UL TPC}

A major feature of LTE is the open-loop UL TPC which is specified in [4]. Apart from the task to upper limit DR, the open-loop UL TPC is also dedicated to control inter-cell interference and to guarantee a certain target signal-tointerference and noise ratio (SINR). A UE's open-loop UL transmit power can be written as [4]

$$
P_{\mathrm{tx}}=\min \left\{P_{\max }, P_{0}+\alpha \cdot L+10 \cdot \log _{10} M+\Delta_{\mathrm{mcs}}\right\},
$$

where $P_{\max }$ is the maximum UE transmit power, $P_{0}$ is a cell/UE specific parameter, $\alpha$ is a cell specific total signal loss correction parameter, $M$ is the number of physical resource blocks (PRBs) assigned to the UE, and $\Delta_{\text {mcs }}$ is a parameter which depends on the Modulation and Coding Scheme (MCS) chosen. $L$ is the total signal loss between transmitter and receiver including path loss, shadowing, fast fading, antenna gain, etc. $L$ is defined as $L=P_{\mathrm{tx}}-P_{\mathrm{rx}}$, where $P_{\text {rx }}$ denotes the receive power. As we are focusing on slow channel variations in this work, we assume that all total signal losses are time-averaged such that fast fading averages out in $L$. Please note that we neglect the closed-loop contribution of the UL TPC, typically denoted as $f\left(\Delta_{i}\right)$ (a UE-specific closed-loop correction factor for short-term corrections, such as interference variations), since this work focuses on slow channel variations (path loss) rather than on fast channel variations such as fast fading. In the following, we consider $\Delta_{\text {mcs }}=0$ in order to maintain focus on parameters of our interest (viz. $\alpha, P_{0}$ ).

By means of the open-loop UL TPC from Eq. (1), it is possible to upper limit DR since differences in the total signal loss $L$ between multiple UEs can be compensated by setting $\alpha>0$. Hence, we are interested on the dependency of the DR as a function of the TPC parameters $\alpha$ and $P_{0}$ given a set $\mathcal{L}$ of the total signal losses that are observed at the BS from all UEs connected to this BS. This dependency has been originally derived in [3]. We summarize the findings in the next subsection.

\subsection{Dynamic range expression}

According to [2] the DR $D$ is defined as the difference between the 5 th and the 95th percentile of the receive powers (in $\mathrm{dB}$ ) of signals that are received at the BS simultaneously, i.e.,

$$
D=Q_{5}\left(\mathcal{P}_{\text {rx }}\right)-Q_{95}\left(\mathcal{P}_{\text {rx }}\right),
$$

where $Q_{x}(\cdot)$ denotes the $x$ th percentile of an ordered set and $\mathcal{P}_{\text {rx }}$ is an ordered set of the observed receive powers in $\mathrm{dB}$. We order the set $\mathcal{P}_{\text {rx }}$ such that $Q_{0}(\cdot)$ addresses the highest value observed and $Q_{100}(\cdot)$ the smallest value observed. In order to exclude extreme outliers, the DR is defined using the difference between the aforementioned percentiles rather than using the difference between maximal and minimal receive power. Please note that DR is specific to a certain cell ${ }^{3}$ because each BS has a different set of observed receive powers $\mathcal{P}_{\text {rx }}$. Nevertheless, we neglect adding a superscript for the cell as this work focuses on an experiment that only involves a single cell. Based on Eq. (2), Berger et al. derive in [3] a closedform expression for DR as a function of the LTE UL TPC parameters $\alpha$ and $P_{0}$ under the assumption that every UE transmits on a single PRB as follows

$$
\begin{aligned}
D & =\min \left(P_{\max }, P_{0}+\alpha L_{\min }\right)-L_{\min } \\
& -\min \left(P_{\max }, P_{0}+\alpha L_{\max }\right)+L_{\text {max }} .
\end{aligned}
$$

In the formula above, $L_{\min }=Q_{5}(\mathcal{L})$ and $L_{\max }=Q_{95}(\mathcal{L})$, where $\mathcal{L}$ denotes the ordered set of observed total signal losses in $\mathrm{dB}$. $\mathcal{L}$ is ordered such that $Q_{0}(\cdot)$ addresses the smallest and $Q_{100}(\cdot)$ the highest total signal loss value observed, respectively ${ }^{4}$. Please note that Eq. (3) enables us setting $\alpha$ and $P_{0}$ such that $D<D_{\mathrm{TH}}$. Generalizing Eq. (3) such that the DR expression considers a realistic scheduler is ongoing work. 
Please note that using Eq. (3) it is possible to obtain both an instantaneous DR if we use an instantaneous set of total signal losses and a time-averaged DR if we use a set of total signal losses which is accumulated over a certain time period. In this work, we focus on a time-averaged DR because we aim to upper limit DR by means of LTE's Open-Loop TPC (OLTPC), which is chosen to be slow ${ }^{5}$. The fastest possible update interval of the OLTPC parameters $\alpha$ and $P_{0}$, given by the LTE standard [7], is $320 \mathrm{~ms}^{6}$. Hence, the set $\mathcal{L}$ shall be obtained by accumulating the observed total signal losses for a minimal time period in the range of seconds. As a consequence, a setting of $\alpha$ and $P_{0}$ which ensures $D \leq D_{\mathrm{TH}}$ according to Eq. (3) is valid on a time average but not necessarily for every instantaneous realization of $\mathcal{L}$. By choosing $\alpha$ and $P_{0}$ such that $D \leq D_{\mathrm{TH}}-\epsilon$ with $\epsilon>0$ we can consider short-term variations. The longer the time period for collecting $\mathcal{L}$, the more we are considering the time-averaged behavior rather than the instantaneous one.

In the following subsection, we explain in detail, why we should upper limit DR and give an estimate on $D_{\mathrm{TH}}$, which is based on the ADC link-budget [3].

\subsection{Dynamic range threshold}

At the $B S$ receiver, the UE separation takes place in the digital domain, i.e., the signals from all UEs received at the same time at the BS must simultaneously pass the analog receiver front-end from antenna to ADC. The analog front-end selects the desired band while suppressing the out-of-band interference, moves it to a low intermediate frequency (low-IF) or baseband and, finally, adapts its amplitude to the requirements of the ADC using the automatic gain control (AGC). The tailored ADC input signal preserves most of the throughput and error rate performance of the signal originally received. As stated in [8], the theoretical DR of an ADC is given by

$$
D_{\mathrm{ADC}}=(6.02 b+4.77)[d B]
$$

where $b$ denotes the effective number of bits (ENOB) available at the ADC. Please note that, $D_{\mathrm{ADC}}$ characterizes the theoretical maximal possible power difference in $\mathrm{dB}$ between two input signals that can be processed simultaneously by the ADC and, therefore, is not equal to the DR $D$ of receive powers which we already introduced. In LTE BSs, the ADC typically has a quantization resolution of 13 bits to 16 bits which corresponds to an ENOB of 11 to 14 bits. Hence, the theoretical DR of a typical ADC equals $D_{\mathrm{ADC}} \approx 70-90 \mathrm{~dB}$, which shall be sufficient to cover the variations of the total signal loss of UEs connected to a typical macro cell (we assume that the total signal loss varies up to $60 \mathrm{~dB}$, but in general it can also be higher). However, the $D_{\mathrm{ADC}}$ which is actually available in real systems is reduced by [3]

- Quantization noise back off (10 dB),

- The required SNR for LTE operation (0 dB, depending on modulation and coding scheme),

- The peak-to-average power ratio (PAPR) of the signals and fast fading $(15 \mathrm{~dB})$,

- Additional limitations of the analog receiver front-end, non-ideal circuit components, and residual interference (15-25 dB).

Taking these reductions into account and considering ADCs with a quantization resolution between 13 and 16 bits, we can estimate the practical maximal possible difference in the power of two signals that can be processed simultaneously by the ADC, denoted as $\tilde{D}_{\mathrm{ADC}}$, to be in the range from 20 to $50 \mathrm{~dB}$ (see Fig. 1). Please note that, $\tilde{D}_{\mathrm{ADC}}=D_{\mathrm{TH}}$.

In the experiment described in the Sections 3 and 4, we aim to measure $D_{\mathrm{TH}}$ for an UL transmission that is very similar to LTE using a 12 bits ADC. Of course, the DR threshold obtained in the following experiment can only be considered as an indicative value similar to the link-budget-based value as the practical hardware implementation may be different and also varies between the BSs (e.g., the ADCs resolution is different). Nevertheless, this measurements help estimating the DR threshold as they are an alternative to the link-budget-based approach and as they can be used to validate the aforementioned approach.

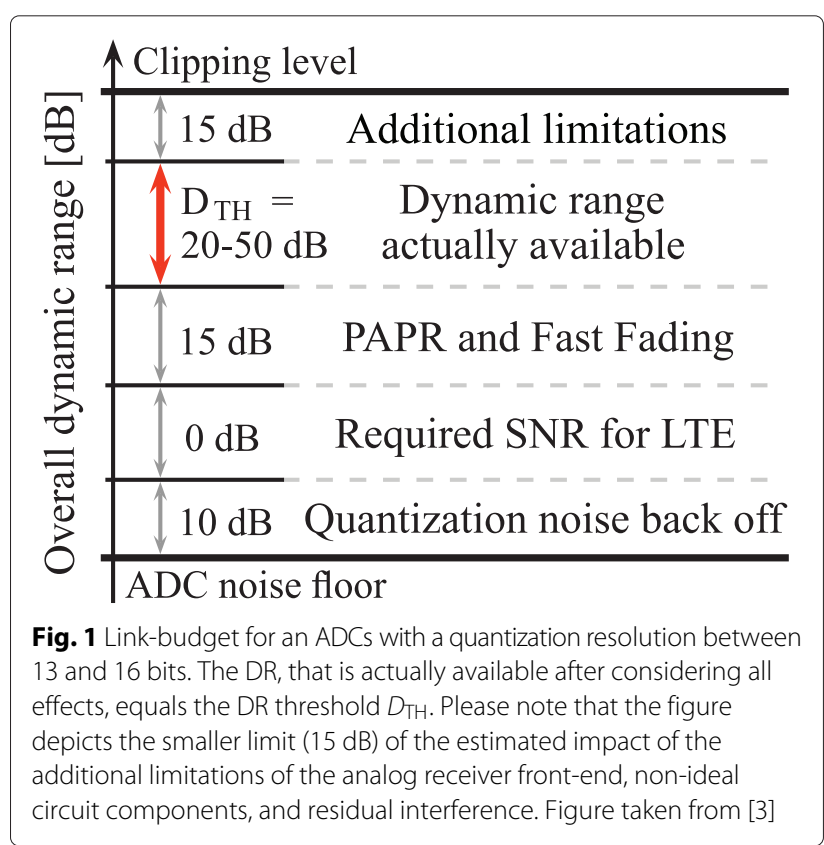




\subsection{Relation to 3GPP}

In [9], Section 7.3, 3GPP also defines a DR. However, 3GPP's DR deviates from the definition used in this work since 3GPP defines DR as

"a measure of the capability of the receiver to receive a wanted signal in the presence of an interfering signal inside the received channel bandwidth",

where the interfering signal is an additive white Gaussian noise (AWGN) signal. In contrast, we consider the interferer to be another UE, i.e., another LTE signal. More related to our work is 3GPP's in-channel selectivity, which is defined in Section 7.4 in [9]. The in-channel selectivity is

"a measure of the receiver's ability to receive a wanted signal at its assigned resource block locations in the presence of an interfering signal received at a larger power spectral density. In this condition a throughput requirement shall be met for a specified reference measurement channel. The interfering signal shall be an E-UTRA ${ }^{7}$ signal [...] and shall be time aligned with the wanted signal".

3GPP connects the in-channel selectivity with a throughput requirement: the throughput in the presence of the interferer shall be $\geq 95 \%$ of the maximal throughput achievable in a reference measurement without the presence of an interferer. This channel-selectivity corresponds to what we define as the DR threshold. However, we have so far defined the DR threshold rather loosely as the DR at which the signal of a weak UE is drown out by the signal of a strong UE, i.e., we have not defined an exact threshold at which we consider a UE to be drowned out. We define this point in Section 5 under consideration of our experimental setup.

Please note that 3GPP also provides requirements of the BS in terms of in-channel selectivity in [9]. For example, the in-channel selectivity shall be better than $21.5 \mathrm{~dB}$ if the wanted signal and the interfering signal are placed right next to each other with each 25 PRBs of bandwidth considering a total channel bandwidth of $20 \mathrm{MHz}$.

\section{Experimental setup}

The experimental setup consists of one evolved NodeB (eNB) and two UEs manufactured by SIGNALION (a National Instruments Company). The setup basically consists of a FPGA-based baseband unit and a radio frontend. Basic operating functionality is provided by the hardware in real-time, including generation of random user data, pilots, and control information at the transmitter side, as well as synchronization and decoding of the control information at the receiver side. Hence, performance metrics such as Received Signal Strength Indicator (RSSI),
Reference Signal Received Power (RSRP), and SINR are available in real time. Further processing of measured data is done with non-real-time scripts using MATLAB. The physical layer is based on LTE release 8 with deviations: In the LTE UL orthogonal frequency division multiplexing (OFDM) is used instead of SC-FDMA. We use OFDMA instead of SC-FDMA since our technical setup does not allow for an SC-FDMA transmission. Nevertheless, we believe that the choice of OFDMA instead of SC-FDMA does not impact the DR threshold measured. The effect that a weak UE is drown out by interference from one or more other UEs in the same cell is caused by the critical receive power difference between the weakest UE and the interferers. However, the only difference in the signal processing at the BS between OFDMA and SC-FDMA is an additional Inverse Discrete Fourier Transform (IDFT), which does not change the power constellation between the weak UE and the interferers. Furthermore, we would like to point out that we can treat the interference of $n$ UEs with each 1 PRB using the SC-FDMA transmission scheme as the same as interference from 1 UE with a bandwidth of $n$ PRBs using the OFDMA transmission scheme as in both cases the receive power probability density function (PDF) over time will be similar. In addition, LTE-Advanced uses clustered SC-FDMA [10] in order to satisfy the demand for flexible UL resource scheduling. This clustered SC-FDMA also has similar PAPR values as OFDMA (dependent on the number of clusters) [10].

During the measurements, the downlink (DL) was transmitted wireless in a laboratory environment. In contrast, the UL signal was transmitted over cables. The reason for realizing the UL using a wire is that we want to ensure stable conditions as we want to measure the exact receive power difference at which the weak UE is drown out. The transmission via wire mitigates all channel effects, i.e., we have no fast fading and no outside interferers. Including the channel effects will not change the fundamental problem in the ADC, however, will make the measurements more unstable and would increase the need for averaging. Please note that a similar wired UL transmission has also been used in [11] and is also proposed in [12]. As the DL transmission is not essential for the measurements carried out in this work, we use a wireless transmission as this transmission form is already realized in the existing experimental setup. The DL transmission needs to be executed for transmitting the control channels. As depicted in Fig. 2, the two UL signals were combined and damped down with a $20 \mathrm{~dB}$ fixed attenuator to prevent clipping. Afterwards, the signal was split again for the eNB receiver and a spectrum analyzer which acts as an observer.

Each UE's transmit power can be regulated. However, the transmit powers have not been calibrated as the experimental setup does not allow for such a measurement. 


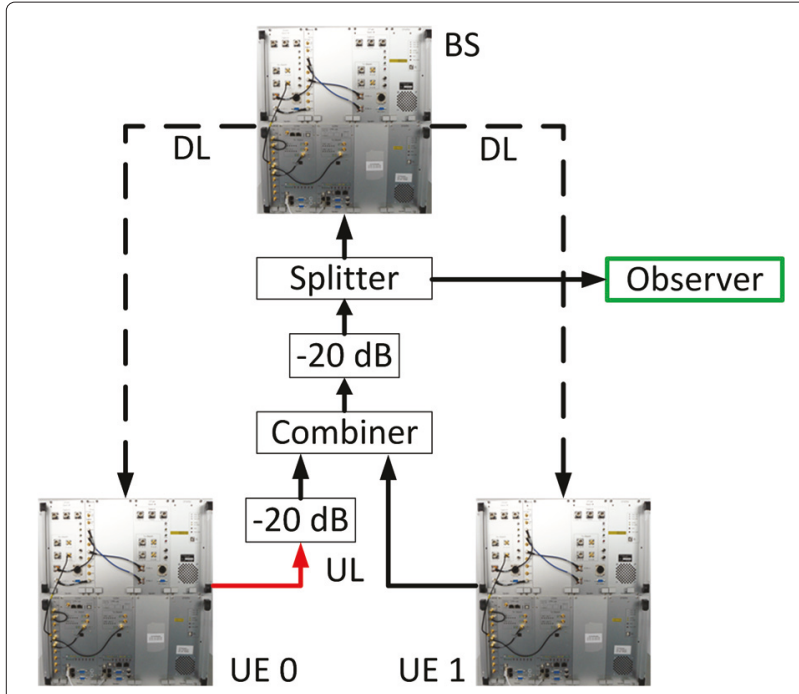

Fig. 2 Schematic view of the measurement setup. The UL transmission (solid lines) is wired, while the DL transmission (dotted lines) is wireless. For both $U L$ and $D L$ transmission, we were using OFDMA. During the experiment, we change the transmit power of UE 0 (indicated by the red solid line) and observe the receive power difference between UE 0 and UE 1 at a spectrum analyzer (indicated by the green box) and the BER at the BS. We use the same hardware for $B S$ and $U E$; however, their firmware is different

Nevertheless, we can change the transmit power of the UEs by switching between predefined power amplifier (PA) transmit power settings, which are denoted by values from $-4 \mathrm{~dB}$ to $-32 \mathrm{~dB}$. The PA transmit power setting of $-4 \mathrm{~dB}$ addresses the highest transmit power possible and equals approximately a total transmit power of $23 \mathrm{dBm}$ at a bandwidth of 30 PRBs. Since a PA transmit power setting ensures a constant transmit power density over the complete bandwidth that is allocated, the total UE transmit power changes when changing the bandwidth of the UE. In order to decrease the receive power of UE 0 even more than it is possible with the transmit power, we add a fixed $20 \mathrm{~dB}$ attenuator for this UE's UL transmission (see Fig. 2).

When performing measurements, the spectrum data and IQ samples received after the ADC at the eNB were stored. The eNB's AGC, which amplifies the complete bandwidth equally, was not fixed and has different values according to the power received at the eNB. After the experiment the Quadrature Phase-Shift Keying (QPSK) constellations, Channel State Information (CSI), and uncoded BER are calculated from the data measured using a script which is compliant to LTE Release 8. In this paper, the BER is used to specify the quality of the link. Table 1 summarizes the relevant configuration parameters.

We would like to point out that an UE's BER depends on the modulation scheme used. In order to remain focus
Table 1 Parameters of the experimental setup

\begin{tabular}{ll}
\hline Common parameters & \\
\hline Bandwidth & $20 \mathrm{MHz}$ \\
FFT size & 2048 \\
Modulation & QPSK \\
UL frequency & $1.99 \mathrm{GHz}$ \\
DL frequency & $2.18 \mathrm{GHz}$ \\
Waveform & OFDM \\
Number of collected TTI & 60 \\
UE 0 & \\
First PRB & 2 \\
Number of allocated PRBs & 30 \\
PA Transmit Power Setting & \\
UE 1 & $-4 \mathrm{to}-32 \mathrm{~dB}$ \\
First PRB & \\
Number of allocated PRBs & 33 \\
PA Transmit Power Setting & $30 / 20 / 10 / 1 / 0$ \\
\hline
\end{tabular}

aWe use values from -4 to $-32 \mathrm{~dB}$ since we cannot precisely calibrate the transmit power using our experimental setup

on the parameters of interest (receive power difference between strong and weak UE), we decided to use QPSK modulation only. Setting the modulation fixed to QPSK is reasonable because the UEs, which have the highest risk of being drown out by other UEs, are the ones at the cell edge. These cell edge UEs will most likely transmit with the most robust modulation scheme they could choose, i.e., with QPSK modulation. Since the BER of the interfering UE 1 is irrelevant for our experiment, we can simply use QPSK modulation for UE 1 as well.

\section{Experimental execution}

Using the setup described in Section 3, we execute the experiment described in the following.

We perform five series of measurements whose configurations are presented in Table 1 . In every series of measurements, we schedule UE 0 with a bandwidth of 30 PRBs, place it at PRB No. 2 in our total channel bandwidth of $20 \mathrm{MHz}$, and schedule UE 1 directly adjacent to UE 0 . The only difference between the five series of measurements is that we change the bandwidth of UE 1 between 30, 20, 10, 1, and 0 PRBs (0 PRBs for UE 1 means that UE 1 does not transmit at all). Figure 3 illustrates the receive power spectrum. We execute the following procedure for all series of measurements. Starting from the initial PA transmit power setting $-4 \mathrm{~dB}$, we incrementally decrease this setting for UE 0 while keeping the PA transmit power setting of $U E 1$ constant at $-4 \mathrm{~dB}$. In this way, the difference in the receive power of UE 0 and UE 1 is successively increased. For every configuration, we measure the receive power density in $\mathrm{ABm} / \mathrm{PRB}$ for both UEs and 


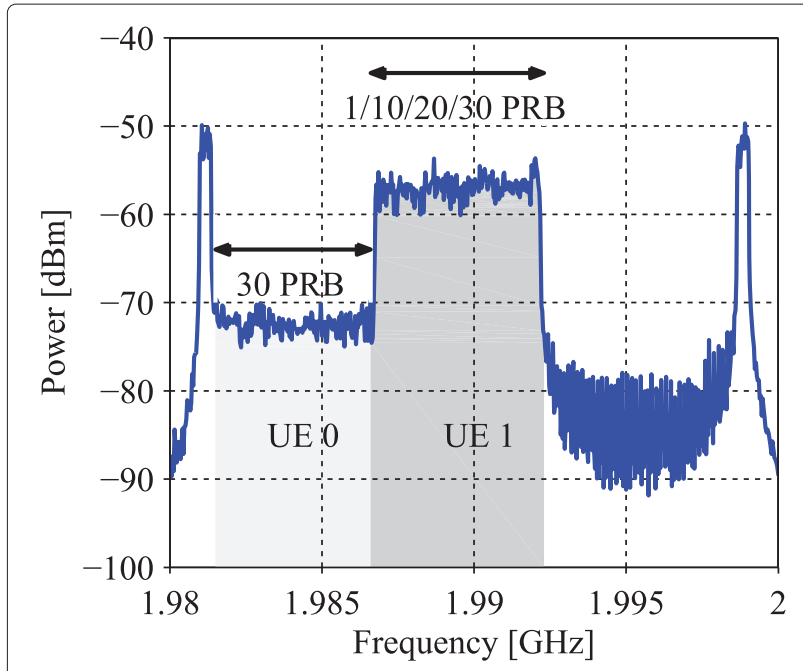

Fig. 3 Illustrative receive power spectrum for the measurement series. UE 1 is always scheduled right next to UE 0 . UE 0 has a fixed bandwidth of 30 PRBs, while the bandwidth of UE 1 is different in every series of measurement. The peaks at the left and right side of the spectrum are control channels. This spectrum is not taken from an actual measurement

compute the BER for UE 0 for a time period of 60 transmission time intervals (TTIs) for each TTI and compute the average of this 60 measurements. The modulation and codings stays constant during the complete experiment.

\section{Evaluation and results}

As mentioned in the previous section, all measurements are averaged over 60 TTIs. In Fig. 4, we present the BER of UE 0 as a function of its receive power density for all five series of measurements. We denote the series of measurements according to the bandwidth of UE 1 except of

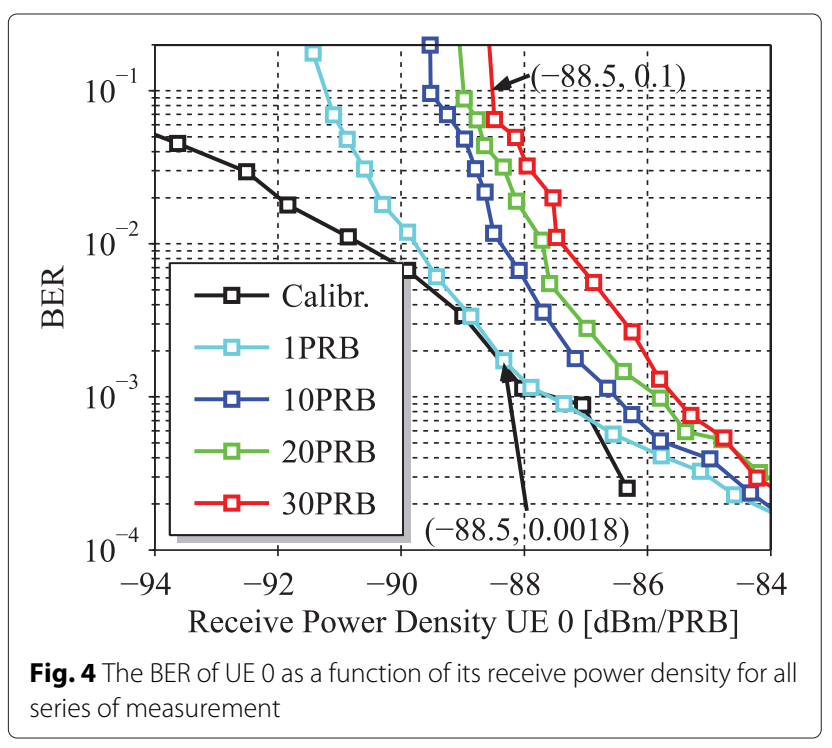

the measurements in which the bandwidth of UE 1 is 0 PRBs. This series of measurements is denoted as calibration (Calibr.). It is visible that the BER strongly increases as the receive power density of UE 0 decreases. Please note that within each series of measurement, the receive power from UE 1 and the noise power stay constant as we decrease the receive power density of UE 0 . However, the series of measurements differ from one other with regard to the receive power of UE 1 . As we are using the same PA transmit power setting, i.e., the same transmit power density, for UE 1 in each series of measurement but modify the bandwidth of UE 1, we also modify the total receive power of UE 1. Furthermore, we can observe that the BER decreases if we reduce the bandwidth of UE 1, i.e., we can farther decrease the receive power of UE 0 until the UE's BER really increases. Comparing the 30 PRB measurement series with the calibration, we can observe that the BERs are considerably lower for the calibration, especially for low receive power densities of UE 0. For example, at a receive power density of $-88.5 \mathrm{dBm} / \mathrm{PRB}$ the $\mathrm{BER}$ is already at 0.1 for the case with UE 1 transmitting on 30 PRBs but only 0.0018 for the case without UE 1 . Hence, we can conclude that the strong BER rise of UE 0 with a decreasing receive power density is mainly caused by the signal of UE 1 and the noise power only plays a minor role, i.e., that the signal of UE 0 is drown out by the strong signal of UE 1 . Of course, when moving towards the measurements with smaller bandwidths for UE 1, the impact of the noise increases. Nevertheless, also in this cases the noise's impact on the BER is small for low receive power densities of UE 0 .

As our measurement setup does not allow us evaluating the data throughput, we have to use a different requirement for the upper limit of DR than 3GPP uses for its in-channel selectivity definition. We decide to consider the signal of UE 0 to be drowned out by the signal of UE 1 if the BER of UE 0 is $\geq 10^{-1}$ since we assume such BER's as a significant performance reduction. We would like to point out that the prior work ([3] and [2]) does not provide a strict measure and/or value to be used for defining the DR threshold. Furthermore, we can observe that the choice of the BER threshold has only a small impact on the resulting DR threshold, as the BER increases rapidly for small receive power densities of UE 0 (see Fig. 4). For example, considering the results for 30 PRBs bandwidth of UE 1, the resulting DR thresholds for critical BERs of 0.05 and 0.2 would be 31.1 and $31.5 \mathrm{~dB}$, respectively. We will see shortly, that this deviation is small compared to the estimated error of the measurement. We compute the DR threshold as follows. For each series of measurement, we obtain the receive power density of UE 0 where its BER equals $10^{-1}$ and compute the corresponding total receive power of UE 0 . Then, we obtain the DR threshold by computing the difference between the aforementioned total 
receive power of UE 0 and the total receive power of $U E 1$. Please note that the total receive power of UE 1 is different for every series of measurement due to the different bandwidth of UE 1. Furthermore, we would like to point out that we consider the DR to be the receive power difference between UE 0 and UE 1, i.e., we use the difference between the maximal and minimal receive power instead of the difference between the 5 th and the 95th percentile of the receive powers (in $\mathrm{dB}$ ) as defined in Eq. (2). The reason for this decision is that we do not need to avoid outliers by means of the percentiles as we only have UE 0 and UE 1 present in our experiment.

In Fig. 5, we present the computed DR thresholds $D_{\mathrm{TH}}$ for all measurements. It is visible that the DR thresholds obtained vary with the series of measurement. $D_{\mathrm{TH}}=$ 31.4 $\mathrm{dB}$ for the $30 \mathrm{PRB}$ measurements while $D_{\mathrm{TH}}=$ 19.4 $\mathrm{dB}$ for the $1 \mathrm{PRB}$ case. If we increase the bandwidth of UE 1, we observe two effects:

1. The total receive power of UE 1 becomes higher, if the receive power density stays constant.

2. The center of interference moves farther away from UE 0 .

Which impact have these effects on the DR threshold measurement? The definition of the DR is related to total power rather than power density (see Eq. (2)). Therefore, effect 1 is inherently included in the computation of the DR threshold. It is clear, that the maximal possible difference in power density must decrease as we increase the bandwidth of UE 1. This is the case in our experiment, as we can see in Fig. 5 (red bars).

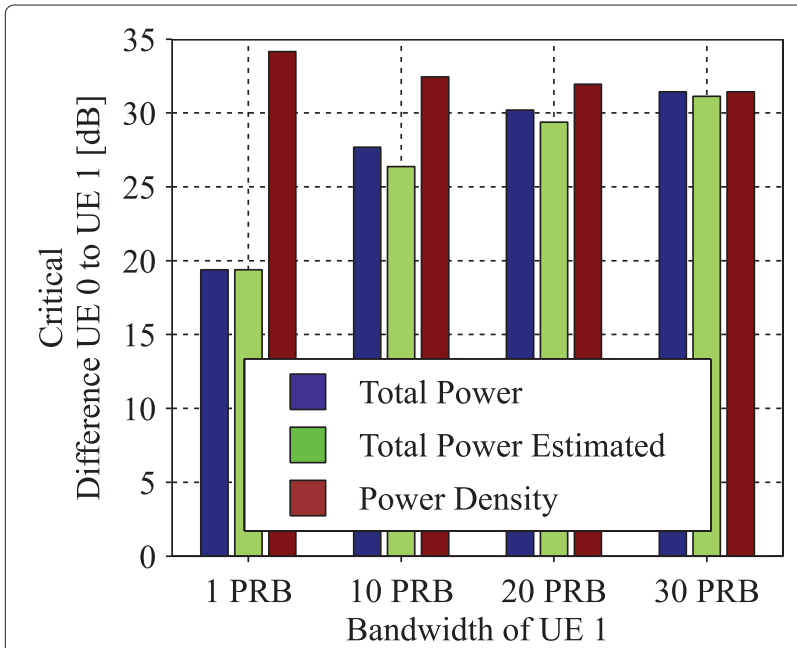

Fig. 5 The critical difference in total power or power density between UE 0 and UE 1 for all bandwidth scenarios. The critical difference is the one at which UE 0 reaches a BER of $10^{-1}$. If we consider the total power, then the critical difference equals the DR threshold
Let us address the second effect mentioned above. Theoretically, the issue of a weak signal being drown out by a strong signal at the ADC conversion is solely impacted by the power difference between these signals. However, as we are defining the weak signal to be drowned out by means of a BER measurement in this experiment, the amount of in-band interference perceived at the weak UE also impacts the result. If we keep the DR between UE 0 and UE 1 constant at a stepwise increasing bandwidth of UE 1, we shift a fraction of the receive power of UE 1 farther away from UE 0 in each step. In this way, the interference perceived at UE 0 decreases because the total power from each sub-carrier of UE 1 decreases with $\frac{1}{f}$ (see sinc-function), where $f$ is the frequency. In the case of $1 \mathrm{PRB}$ at UE 1 , all the power of UE 1 is located in the 12 sub-carriers right next to UE 0 and, hence, the inband interference at UE 0 is much higher than for the case where the said sub-carriers next to UE 0 have less power as the remaining power of UE 1 is located in the additional PRBs farther away from UE 0 (e.g., when UE 1 has 30 PRBs). Following this line of thinking, we can estimate the interference reduction at UE 0 as we increase the bandwidth of UE 1 while keeping the DR constant, i.e., while keeping the total receive power difference between UE 0 and UE 1 constant. Taking the scenario with 1 PRB bandwidth at UE 1 as reference, we know that the center of interference, i.e., the power center of UE 1, moves away from UE 0 by 5,10 , and 15 PRBs for the scenarios where UE 1 has 10, 20, and 30 PRBs, respectively. Hence, considering an in-band power decay of $\frac{1}{f}$, we can estimate that the interference UE 0 perceives from UE 1 decreases by 7,10 , and $11.8 \mathrm{~dB}$ compared to the setting where UE 1 has 1 PRB bandwidth when increasing the bandwidth of UE 1 to 10, 20, and 30 PRBs, respectively ${ }^{8}$. In Fig. 5, we present how the DR threshold is expected to change with respect to the reference measurement series with 1 PRB bandwidth of UE 1 using green bars. The results actually measured are depicted in blue bars. It is visible that measurement and estimation fit well, which supports the statement that the changing result of the DR threshold is caused by the varying interference scenario. We would like to emphasize again, that we believe that in theory the inband interference scenario does not impact the effect of a weak signal being drown out by a strong signal in the ADC because this is solely impacted by the power difference of both signals. However, since we are forced to detect the status of a signal being drown out by means of a BER measurement, the interference situation impacts our measurement result.

Error analysis Please note that, our experimental results on the DR threshold will change only slightly as we change the BER value at which we consider UE 0 to be drowned out by the signal from UE 1 since the increase of the 
BER is very steep for small receive power densities of UE 0 (see Fig. 4). This fact has already been addressed above. Hence, we can observe that our DR threshold result is more sensitive to measurement errors of the receive power rather than on the BER. In order to estimate the error arising from the erroneous receive power measurements, we compute the standard deviation $\sigma_{\mathrm{P}_{\mathrm{rx}}}$ of the measured total receive power, which is Gaussian distributed, at constant transmit power. $\sigma_{\mathrm{P}_{\mathrm{rx}}}=1.9 \mathrm{~dB}$ and represents our random errors. We neglect the systematic error, since the DR computation is actually a difference measurement which eliminates the majority of the systematic error. Using the law of propagation of uncertainty with a double standard deviation in order to achieve $95 \%$ confidence level, we can compute the error of the DR threshold arising from the receive power measurements as $\Delta_{\mathrm{D}_{\mathrm{TH}}, \mathrm{P}_{\mathrm{rx}}}=2 \cdot 1.9 \mathrm{~dB}+2 \cdot 1.9 \mathrm{~dB}=7.6 \mathrm{~dB}$, i.e., $D_{\mathrm{TH}}=31.4 \pm 7.6 \mathrm{~dB}$ for the measurement series with 30 PRBs at UE 1 .

Comparison between measurement and link-budgetbased estimation For the comparison with the linkbudget-based estimation, we use the measurement series in which UE 1 has 30 PRBs of bandwidth. As already discussed above, the dependency of the DR threshold on the bandwidth of UE 1 is an unwanted effected arising from a varying in-band interference scenario in each series of measurement. This effect cannot be avoided in our experiment as we need to declare UE 0 to be drowned out by means of a BER measurement. Hence, when comparing our result to the one from [3], we shall use the series of measurement in which UE 0 perceives the smallest in-band interference which is the case in the series of measurement where UE 1 has 30 PRBs of bandwidth.

Since the quantization resolution of the ADC employed in this experiment was only 12 bits, the ADC's theoretical $\mathrm{DR} D_{\mathrm{ADC}} \approx 65 \mathrm{~dB}$. Following the link-budget calculations from Section 2, we subtract $10 \mathrm{~dB}$ for quantization noise back off, $5 \mathrm{~dB}$ for PAPR [13], and 15-25 dB for additional limitations of the analog receiver front-end, etc ${ }^{9}$. Hence, we would estimate the DR threshold for our setup to be $\tilde{D}_{\mathrm{ADC}}=D_{\mathrm{TH}}=25-35 \mathrm{~dB}$. We can write:

$$
\begin{aligned}
\text { Measurement: } D_{\mathrm{TH}} & =23.8-39.0[\mathrm{~dB}] \\
\text { Link-Budget: } D_{\mathrm{TH}} & =25-35[\mathrm{~dB}] .
\end{aligned}
$$

Hence, we conclude that the link-budget-based estimation is quite accurate.

Impact of further UEs In theory, the presence of further UEs does not change the DR threshold of a receiver, since the DR threshold is solely given by the maximal possible difference between strong and weak receive powers but is independent of the number of UEs received. Nevertheless, in our experiment, we would expect a slight degradation of the DR thresholds measured, if we add further UEs, e.g., at the remaining free bandwidth to the right of UE 1 (see Fig. 3). The additional UEs would increase the inband interference perceived at UE 0 which will increase its BER and, hence, decrease the DR threshold measured. However, since these additional UEs are rather far away from UE 1, we would expect only very minor changes of the resulting DR threshold.

\section{Conclusions}

The uplink dynamic range threshold has been measured to be $D_{\mathrm{TH}}=31.4 \pm 7.6 \mathrm{~dB}$ for a LTE-like measurement setup using an analog-to-digital converter with 12 bits quantization resolution. This result coincides with the link-budget-based estimation from our previous work [3].

We can conclude that the link-budget-based estimation from [3] is an adequate method for obtaining the uplink dynamic range threshold for the receiver at hand. Furthermore, we conclude that the uplink dynamic range plays an important role when configuring the uplink transmit power control since the differences in total signal losses between users connected to the same base station can easily be larger than or equal to $60 \mathrm{~dB}$. This holds true not only for LTE but also for any future systems in which the user separation takes place after the analog-to-digital converter. We would like to point out that the problem of limiting the uplink dynamic range can be considered to become more challenging in future due to the arising trend of receiving more and more signals and bandwidth simultaneously using one analog-to-digital converter (see, e.g., carrier aggregation). Also, we would like to mention that in practical scenarios users which are at the cell edge are typically transmitting on only a couple of PRBs because they are not capable transmitting on more PRBs, while users with a good channel may have considerably more bandwidth. This bandwidth differences, in fact, provoke a high dynamic range.

Ongoing work is focusing on the impact of the scheduler on the uplink dynamic receive power range.

\section{Endnotes}

${ }^{1}$ Please note that we neglect the weakest $5 \%$ of the UEs in the DR definition in order to avoid large DR's due to outliers.

${ }^{2}$ In nowadays LTE networks ADCs have a typical resolution of 16 bits [5, 6].

${ }^{3} \mathrm{~A}$ cell is the area covered by one sector. Depending on the sectorization a BS might consist of multiple sectors.

${ }^{4}$ Please note that, the sets $\mathcal{P}_{\text {rx }}$ and $\mathcal{L}$ are ordered differently in order to address the fact that large receive powers are created by small path losses.

${ }^{5}$ Slow means that the temporal granularity of the OLTPC is much larger than the fast fading varies. 
${ }^{6}$ Note that in LTE adaptive modulation and coding is chosen to follow the fast term channel variations.

${ }^{7}$ evolved UMTS Terrestrial Radio Access

${ }^{8} 10 \log \left(\frac{1}{5}\right) \approx-7 \mathrm{~dB}, 10 \log \left(\frac{1}{10}\right) \approx-10 \mathrm{~dB}$,

$10 \log \left(\frac{1}{15}\right) \approx-11.8 \mathrm{~dB}$

${ }^{9}$ In contrast to the link-budget in Section 2, we do not subtract $10 \mathrm{~dB}$ for fast fading because the UL transmission has been realized via cable.

\section{Competing interests}

The authors declare that they have no competing interests.

\section{Author details}

${ }^{1}$ Vodafone Chair Mobile Communcations Systems, Technische Universität Dresden, 01062 Dresden, Germany. ${ }^{2}$ Nokia Networks, St.-Martin-Straße 76, 81541 Munich, Germany. ${ }^{3}$ Nomor Research, Brecherspitzstraße 8, 81541

Munich, Germany.

Received: 15 December 2014 Accepted: 11 September 2015

Published online: 06 October 2015

\section{References}

1. L Smaini, Rf analog impairments modeling for communication systems simulation: application to OFDM-based transceivers. (John Wiley and Sons, Ltd, Chichester, UK, 2012), pp. 199-202

2. O Bulakci, S Redana, Impact of power control optimization on the system performance of relay based LTE-Advanced heterogeneous networks. J. Commun. Netw. 13(4), 345-359 (2011)

3. S Berger, B Almeroth, V Suryaprakash, P Zanier, I Viering, G Fettweis, Dynamic Range-Aware Uplink Transmit Power Control in LTE Networks: Establishing an Operational Range for LTE's Open-Loop Transmit Power Control Parameters ( $\alpha$,P0). IEEE Wirel. Commun. Lett. 3(5), 521-524 (2014)

4. 3GPP Technical Specification Group Radio Access Network: TS 36.213; E-UTRA Physical layer procedures. Tech. Rep. (2014)

5. Texas Instruments: 16-Bit. 80/105/135-MSPS Analog-to-Digital Converters (2009). http://www.ti.com.cn/cn/lit/ds/symlink/ads5482.pdf Accessed 2015-04-22

6. Linear Technology: Wireless \& RF Solutions; High Performance Analog ICs. (2011). http://cds.linear.com/docs/en/solutions-manual/ Wireless_and_RF_Solutions.pdf Accessed 2015-04-22

7. 3GPP Technical Specification Group Radio Access Network: TS 36.331; E-UTRA Radio Resource Control (RRC) Protocol specification. Tech. Rep. (2014)

8. JH Reed, Software radio: a modern approach to radio engineering. (Prentice Hall, New Jersey, 2002)

9. 3GPP Technical Specification Group Radio Access Network: TS 36.104; E-UTRA Base Station (BS) radio transmission and reception (Release 12). Tech. Rep. (2014)

10. M. Rumney (ed.), LTE and the Evolution to 4G Wireless: Design and Measurement Challenges, 2nd edn. (Wiley, Chichester, UK)

11. M Danneberg, R Datta, A Festag, G Fettweis, in Sensor Array and Multichannel Signal Processing Workshop (SAM), 2014 IEEE 8th. Experimental testbed for $5 \mathrm{G}$ cognitive radio access in $4 \mathrm{G}$ LTE cellular systems, (2014), pp. 321-324

12. RF Test and Measurement. Fig. A.4. http://www.sharetechnote.com/html/ RF Measurement_LTE.html Accessed 2015-04-20

13. N Soltani, Comparison of single-carrier FDMA vs. OFDMA as $3 g p p$ long-term evolution uplink. EE359 Project (2009)

\section{Submit your manuscript to a SpringerOpen ${ }^{\circ}$ journal and benefit from:}

- Convenient online submission

- Rigorous peer review

- Immediate publication on acceptance

- Open access: articles freely available online

- High visibility within the field

- Retaining the copyright to your article

Submit your next manuscript at $>$ springeropen.com 